\title{
Farmacocinética de vancomicina en niños hospitalizados en una unidad de paciente crítico
}

\author{
Carlos Acuña, Jorge Morales, Cecilia Castillo y Juan P. Torres
}

\section{Phamacokinetics of vancomycin in children hospitalized in a critical care unit}

Introduction: Monitoring PK/PD of vancomycin with basal and peak serum levels and the area under the curve of drug exposure $24 \mathrm{~h} / \mathrm{MIC}$ (ABC $24 \mathrm{~h} / \mathrm{MIC}$ ) could optimize the management of children. Objective: To study the PK of vancomycin in children hospitalized in the Pediatric Intensive Care Unit (PICU), assessing PK/PD parameters withABC24 h/MIC. Methods: Retrospective, descriptive study in the PICU (Hospital Luis Calvo Mackenna) between January 2008-March 2010. We included children $<18$ years who required antimicrobial treatment with vancomycin for suspected/confirmed staphylococcal infection using a dose of $40 \mathrm{mg} / \mathrm{k} /$ day. Plasmatic levels were performed one hour postinfusion and 30 min prior to the next dose. The following PK/PD parameters were calculated: vancomycin clearance, elimination rate constant, volume of distribution, half-life (T1/2) and ABC $24 \mathrm{~h} /$ MIC. Results: We enrolled eighty-four children. According to ABC 24 h/MIC obtained, 54\% (45/84) of children reached an optimal level ( $>400 \mathrm{mg} * \mathrm{hr} / \mathrm{L})$. Based on the traditional PK/PD parameters, $49 \%$ of cases $(41 / 84)$ presented a basal level of vancomycin in the therapeutic range $(5-15 \mu \mathrm{g} / \mathrm{mL})$ and of those, only $39 \%(16 / 41)$ had a ABC 24 h/MIC over $400 \mathrm{mg}$ *h/L. Discussion: Based on our results, children admitted to PICU could be exposed to sub therapeutic doses of vancomycin. We recommend to implement tailored antimicrobial treatment monitoring vancomycin PK/PD parameters.

Key words: Vancomycin, pharmacokinetics, sepsis, children.

Palabras clave: Vancomicina, farmacocinética, sepsis, niños.

\section{Introducción}

$\mathrm{E}$ 1 shock séptico en pediatría se asocia a la liberación de mediadores inflamatorios e hipovolemia secundaria grave que requiere administración enérgica de volumen ${ }^{1}$, lo cual contribuye a variaciones importantes en el volumen de distribución ( $\mathrm{Vd}$ ) de los fármacos en niños ${ }^{2,3}$. Para un adecuado uso de antimicrobianos en infecciones graves se deben considerar las variaciones de parámetros farmacocinéticos y farmacodinámicos $(\mathrm{PK} /$ PD) debido al estado de inflamación sistémica (en inglés systemic inflammatory response syndrome- SIRS $)^{4,5}$.

Los parámetros PK/PD más relevantes para el uso de antimicrobianos que relacionan el tiempo de exposición del fármaco y su efecto farmacológico, son: el tiempo en que la concentración plasmática está sobre la concentración inhibitoria mínima del microorganismo ( $\mathrm{T}>\mathrm{CIM}$ ), el valor en que la concentración máxima o pico se mantiene sobre la CIM del microorganismo (Cmax/CIM) y el área bajo la curva de exposición del fármaco en $24 \mathrm{~h}$ sobre la CIM (ABC 24 h/CIM) $)^{6-8}$.

Existen estudios in vitro e in vivo con vancomicina donde se han comparado estos parámetros con la eficacia clínica y microbiológica, recomendándose utilizar $\mathrm{ABC}$ $24 \mathrm{~h} / \mathrm{CIM}$ como una herramienta válida para estimar el éxito clínico y microbiológico del tratamiento antimicrobiano $^{9-11}$. Moise-Broder y cols. ${ }^{12}$, demostraron en 160 adultos con infección respiratoria baja causada por Staphylococcus aureus que la respuesta clínica y microbiológica a vancomicina fue superior en pacientes con mayor ABC 24 h/CIM, sin encontrar relación con el porcentaje de tiempo en que la concentración plasmática de vancomicina se mantuvo sobre la CIM. El mismo grupo ${ }^{13}$, describió el uso de ABC 24 h/CIM para estimar el éxito clínico y microbiológico del tratamiento de la neumonía asociada a ventilación mecánica por $S$. aureus, mostrando que un $\mathrm{ABC} 24 \mathrm{~h} / \mathrm{CIM}>345 \mathrm{mg} * \mathrm{~h} / \mathrm{L}$ se relacionó con un mejor éxito clínico y cuando fue $>850 \mathrm{mg} * \mathrm{~h} / \mathrm{L}$, con un mejor éxito microbiológico ${ }^{14,15}$.

En pediatría, y más aún en niños con patologías críticas, no se conoce si los modelos PK/PD descritos en adultos ofrecen ventajas farmacológicas al usar ABC 24 $\mathrm{h} / \mathrm{CIM}>400 \mathrm{mg} * \mathrm{~h} / \mathrm{L}$ para obtener un mejor éxito clínico y microbiológico en el tratamiento de las infecciones causadas por $S$. aureus ${ }^{17}$. Un estudio realizado por Giachetto y cols. ${ }^{17}$, describió 23 pacientes pediátricos que ingresaron a la Unidad de Paciente Crítico Pediátrico (UPCP) en los que los parámetros $\mathrm{PK} / \mathrm{PD}$ fueron muy variables y sólo un grupo menor de pacientes tuvo ABC $24 \mathrm{~h} / \mathrm{CIM}$ en un rango óptimo para efectividad terapéutica. Con estos
Hospital Luis Calvo Mackenna, Santiago, Chile.

Unidad de Paciente Crítico (CA, CC) Unidad de Farmacia Clínica (JM). Facultad de Medicina, Universidad de Chile, Santiago.

Departamento de Pediatría y Cirugía Infantil Oriente (JPT).

Los autores declaran no tener conflictos de interés

Este trabajo no contó con fuentes de financiamiento

Recibido: 24 de abril de 2013 Aceptado: 5 de septiembre de 2013

Correspondencia a: Jorge Morales Vallespín farmaciaclinica@calvomackenna.cl 
antecedentes, parece razonable considerar la medición de concentraciones plasmáticas de vancomicina en esta población.

El objetivo de este estudio fue determinar los parámetros farmacocinéticos y el ABC 24 h/CIM en niños ingresados en la UPCP que utilizaron dosis habituales de $40 \mathrm{mg} / \mathrm{kg} /$ día de vancomicina y evaluar si existen diferencias en los parámetros farmacocinéticos por edad en los pacientes pediátricos $\geq$ dos años $\mathrm{y}<$ dos años.

\section{Pacientes y Métodos}

Se realizó un estudio retrospectivo, descriptivo en la UPCP del Hospital Luis Calvo Mackenna en Santiago, Chile entre enero del año 2008 y marzo de 2010. Se incluyeron pacientes $<18$ años que requirieron tratamiento antimicrobiano con vancomicina (Hospira ${ }^{\circledR}$, Suiza) por sospecha o confirmación de infección por $S$. aureus y recibieron dosis de $10 \mathrm{mg} / \mathrm{kg}$ cada $6 \mathrm{~h}$ (40 mg/kg/día) en las primeras $24 \mathrm{~h}$ de su ingreso a la UPCP. Se excluyeron aquellos pacientes que recibieron dosis de carga de $15 \mathrm{mg} /$ $\mathrm{kg}$ de vancomicina, los que fueron sometidos a terapia de reemplazo renal (hemo/peritoneo-diálisis) y los pacientes con antecedente de alergia a vancomicina. El estudio fue aprobado por el Comité de Ética Científico Pediátrico del Servicio de Salud Metropolitano Oriente.

Los pacientes enrolados recibieron a la hora cero una infusión de vancomicina intravenosa durante 60 minutos, que fue repetida cada $6 \mathrm{~h}$. Se realizó medición de concentraciones plasmáticas de vancomicina en $1 \mathrm{~mL}$ de sangre

Tabla 1. Parámetros PK/PD de acuerdo al grupo etario en niños hospitalizados en una Unidad de Paciente Crítico Pediátrico que recibieron tratamiento con vancomicina

\begin{tabular}{|c|c|c|c|}
\hline Parámetro PK/PD & < 2 años (n: 47) & $\geq 2$ años (n: 34) & $\mathbf{p}$ \\
\hline Peso kg (mediana, p25-75) & $6,5(5-8)$ & $26(17-36)$ & $<0,001$ \\
\hline $\begin{array}{l}\text { Dosis de vancomicina mg/kg/día } \\
\text { (mediana, p25-75) }\end{array}$ & $40(40-40)$ & $40(40-40)$ & 0,09 \\
\hline T 1/2 (h) (mediana, p25-75) & $3,6(2,2-5,5)$ & $3,8(2,7-10,6)$ & 0,18 \\
\hline Ke (L/h) (mediana, p25-75) & $0,19(0,12-0,3)$ & $0,18(0,06-0,25)$ & 0,18 \\
\hline VD (L/Kg) & $0,54(0,42-0,89)$ & $0,62(0,41-1,04)$ & 0,34 \\
\hline $\begin{array}{l}\text { Cl Vancomicina (L/h) (mediana, p25- } \\
\text { 75) }\end{array}$ & $0,67(0,39-1,15)$ & $2,63(1,53-3,73)$ & $<0,001$ \\
\hline $\begin{array}{l}\text { Niveles basales de vancomicina } \\
(\mu \mathrm{g} / \mathrm{mL})(\text { mediana, p25-75) }\end{array}$ & $11,04(5,9-17,6)$ & $11,43(5,6-22,5)$ & 0,45 \\
\hline $\begin{array}{l}\text { Niveles pico de vancomicina } \\
(\mu \mathrm{g} / \mathrm{mL})(\text { mediana, p25-75) }\end{array}$ & $24,2(15,7-32,3)$ & $25,4(17,3-36,7)$ & 0,73 \\
\hline $\begin{array}{l}\text { ABC } 24 \text { h/CIM }\left(\mathrm{mg}^{*} \mathrm{~h} / \mathrm{L}\right) \\
\text { (mediana, p25-75) }\end{array}$ & $430,7(242,2-612,7)$ & $410,6(261,5-689,1)$ & 0,63 \\
\hline \multicolumn{4}{|c|}{$\begin{array}{l}\mathrm{T}_{1 / 2} \text { : tiempo de vida media de vancomicina; Ke: constante de eliminación; VD: volumen de distribución; } \\
\mathrm{Cl} \text { : clearance; } \mathrm{ABC} 24 \mathrm{~h} / \mathrm{CIM} \text { : área bajo la curva de exposición del fármaco en } 24 \mathrm{~h} \text { sobre la concen- } \\
\text { tración inhibitoria mínima }(\mathrm{CIM}) \text {. }\end{array}$} \\
\hline
\end{tabular}

sin centrifugar, una hora post-infusión (nivel pico, valor de referencia $25-40 \mu \mathrm{g} / \mathrm{mL}$ ) y 30 min antes de la siguiente dosis (nivel basal, valor de referencia $5-15 \mu \mathrm{g} / \mathrm{mL}$ ), una vez que se cumplieron 5-7 vidas medias del fármaco (48 $\mathrm{h}$ luego del inicio del tratamiento con vancomicina). Para la medición de las concentraciones plasmática se utilizó la técnica de inmunoensayo de polarización de fluorescencia (TDxFLx, Abbott Laboratories, IL, EE.UU.), de acuerdo a las instrucciones del fabricante.

\section{Análisis del modelo farmacocinético}

El modelo mono-compartimental fue utilizado para el seguimiento farmacocinético el que consistió en la medición de concentraciones plasmática basales y pico de vancomicina una vez que se alcanzó el estado de equilibrio. Los parámetros farmacocinéticos se calcularon utilizando métodos bayesianos con los software Therapeutic Drug Monitoring System 2000 y LEXI calc, determinándose el clearance de vancomicina $(\mathrm{Cl}$ vancomicina), la constante de eliminación (ke), el volumen de distribución ( $\mathrm{Vd})$, la vida media $\left(\mathrm{T}_{1 / 2}\right)$ y ABC $24 \mathrm{~h} / \mathrm{CIM}$ (valor de referencia $\geq 400 \mathrm{mg} * \mathrm{~h} / \mathrm{L}$ y considerando CIM de $1 \mu \mathrm{g} / \mathrm{mL}$ para $S$. aureus).

\section{Análisis estadístico}

La comparación de las variables entre grupos se realizó por prueba de T-Student o prueba de Mann-Whitney, de acuerdo a la distribución de los datos. Se utilizó $\chi^{2}$ con corrección de Yates o prueba exacta de Fisher para la asociación entre variables categóricas. Los cálculos de parámetros farmacocinéticos se realizaron con el software de farmacocinética Therapeutic Drug Monitoring System 2000. La significancia estadística utilizada fue de $\mathrm{p}<0,05$ y para todos los análisis estadísticos se utilizó el programa Sigma Stat 3.0 (SPSS Science, Chicago, IL).

\section{Resultados}

Se enrolaron 84 pacientes tratados con vancomicina, de los cuales 43 fueron varones, con un promedio de edad de 4,6 años (rango 0-16). La patología de base de los pacientes fue: $19 \%$ sospecha de endocarditis, $17 \%$ tuvo un trasplante hepático/renal, 15\% presentó un episodio de neutropenia febril, $10 \%$ desarrolló un shock séptico, $8 \%$ tuvo neumonía y $6 \%$ correspondió a pacientes quemados graves.

\section{Parámetros farmacocinéticos comparando grupos etarios}

Las concentraciones séricas de vancomicina, los parámetros farmacocinéticos y ABC 24 h/CIM de niños $<2$ años y $\geq 2$ años de edad se muestran en la Tabla 1.

La mediana de la dosis indicada de vancomicina fue de $40 \mathrm{mg} / \mathrm{kg} /$ día. Los volúmenes de distribución (VD) 
$<2$ años y $\geq 2$ años fueron similares $(0,54 \mathrm{~L} / \mathrm{kg}$ versus $0,62 \mathrm{~L} / \mathrm{kg}$, respectivamente; $\mathrm{p}: 0,34)$. La vida media $\left(\mathrm{T}_{1 / 2}\right)$ y la constante de eliminación (Ke) entre ambos grupos etarios no presentó diferencias significativas (p: 0,18 en ambas comparaciones).

El clearance de vancomicina ( $\mathrm{Cl}$ vancomicina) fue significativamente mayor en los niños $>$ dos años $(2,63$ $\mathrm{L} / \mathrm{h}$ versus $0,67 \mathrm{~L} / \mathrm{h} ; \mathrm{p}<0,001)$.

No se observaron diferencias significativas al comparar los dos grupos etarios en relación a las concentraciones plasmáticas basales, pico y ABC $24 \mathrm{~h} / \mathrm{CIM}$ de vancomicina.

\section{ABC 24 h/CIM en relación a parámetros PK/PD tradicionales}

De acuerdo con el ABC $24 \mathrm{~h} / \mathrm{CIM}$ obtenido, 54\% (45/84) de los niños alcanzó un nivel óptimo deseado $(>400 \mathrm{mg} * \mathrm{~h} / \mathrm{L})$. De los casos que presentaron un ABC 24 h/CIM no óptimo (39/84; 46\%), 26 de ellos (66\%) tuvieron concentraciones plasmáticas basales de vancomicina en rango terapéutico $(5-15 \mu \mathrm{g} / \mathrm{mL})^{18}$ según los parámetros farmacocinéticos tradicionales. Según el análisis por parámetros farmacocinéticos tradicionales, $49 \%$ de los casos (41/84) tuvo concentraciones plasmáticas basales de vancomicina en rango terapéutico $(5-15 \mu \mathrm{g} / \mathrm{mL})^{18}$ y de ellos, sólo 39\% (16/41) presentó un ABC 24 h/CIM mayor a $400 \mathrm{mg} * \mathrm{~h} / \mathrm{L}$. De esta manera, 61\% (25/41) de los pacientes no alcanzó un nivel óptimo deseado de $\mathrm{ABC}$ 24 h/CIM (Figura 1).

Al estudiar aquellos pacientes con concentraciones plasmáticas basales de vancomicina entre 5,0 y $9,9 \mu \mathrm{g} / \mathrm{mL}$, no alcanzaron una ABC $24 \mathrm{~h} / \mathrm{CIM}>400 \mathrm{mg} * \mathrm{~h} / \mathrm{L}$ el 84\% de los casos. Por otra parte, aquellos con concentraciones plasmáticas de vancomicina basales entre 10,0 a 14,9 $\mu \mathrm{g} /$ $\mathrm{mL}$, en $81 \%$ de los casos tuvieron una ABC $24 \mathrm{~h} / \mathrm{CIM}$ $>400 \mathrm{mg} * \mathrm{~h} / \mathrm{L}(\mathrm{p}<0,0001)$.

\section{ABC 24 h/CIM en relación a parámetros farmacocinéticos según grupo etario}

Al comparar ABC $24 \mathrm{~h} / \mathrm{CIM}$ versus parámetros farmacocinéticos según grupo etario $(<2$ años $\mathrm{y} \geq 2$ años $)$ (Figura 2), destaca la correlación directamente proporcional entre concentraciones plasmáticas de vancomicina y ABC $24 \mathrm{~h} / \mathrm{CIM}$ en pacientes $<2$ años con un $\mathrm{R}^{2}=0,85 \mathrm{y}$ entre concentraciones pico de vancomicina y ABC $24 \mathrm{~h} /$ CIM en $<2$ años, con un $\mathrm{R}^{2}=0,85$.

\section{Discusión}

En el presente estudio, los parámetros farmacocinéticos tradicionales (VD, T1/2 y concentraciones plasmáticas basal y pico) estuvieron dentro de rangos terapéuticos en niños ingresados a la UPCP y que recibieron una dosis de vancomicina (mediana) de $40 \mathrm{mg} / \mathrm{kg} /$ día. Sin

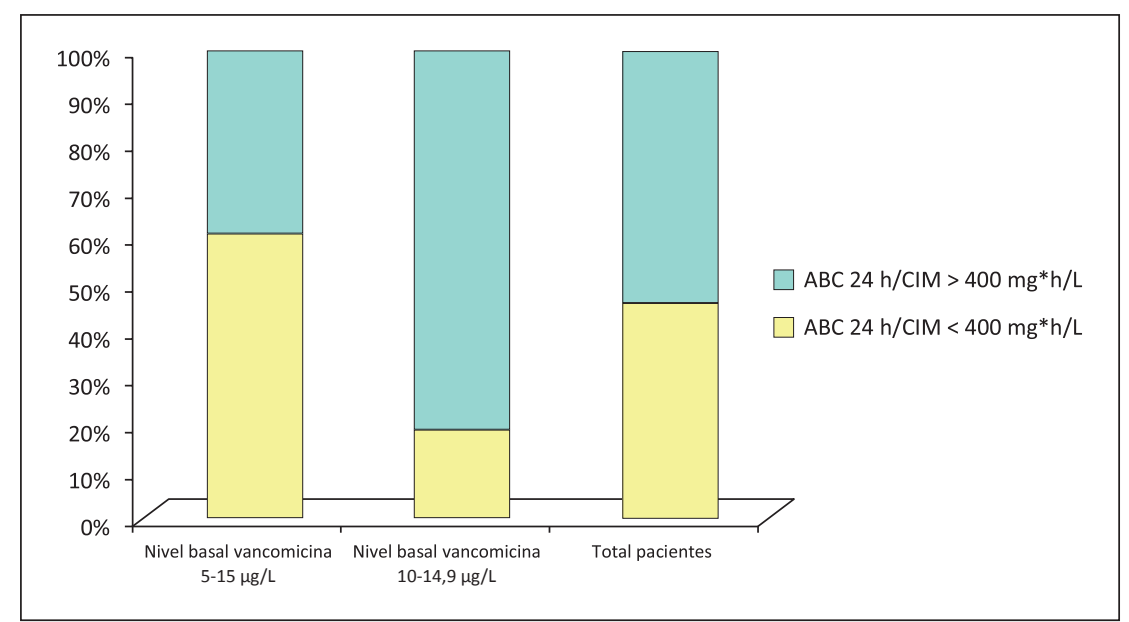

Figura 1. Área bajo la curva de exposición del fármaco en 24 h sobre la concentración inhibitoria mínima ( $A B C 24$ h/CIM) en relación a parámetros PK/PD tradicionales (concentraciones plasmáticas basales y pico de vancomicina) en niños hospitalizados en una Unidad de Paciente Crítico Pediátrico que recibieron tratamiento con vancomicina.

embargo, el análisis de ABC 24 h/CIM mostró que sólo $54 \%$ de los niños alcanzó un valor óptimo deseado de $400 \mathrm{mg} * \mathrm{~h} / \mathrm{L}$. Además, se demostraron diferencias significativamente menores en los valores de ABC $24 \mathrm{~h} / \mathrm{CIM}$ cuando existieron concentraciones plasmáticas basales de vancomicina entre 5,0 y $9,9 \mu \mathrm{g} / \mathrm{mL}$, comparado con niños con concentraciones basales de $10-15 \mu \mathrm{g} / \mathrm{mL}^{18}$ ( $\mathrm{p}<0,0001)$. Con concentraciones plasmáticas basales de vancomicina de 5,0 a $9,9 \mu \mathrm{g} / \mathrm{mL}, 84 \%$ de los pacientes tuvo una $\mathrm{ABC} 24 \mathrm{~h} / \mathrm{CIM}<400 \mathrm{mg} * \mathrm{~h} / \mathrm{L}$, mientras que niños con concentraciones basales de 10-15 $\mu \mathrm{g} / \mathrm{mL}$, sólo $16 \%$ de los pacientes tuvo ABC $24 \mathrm{~h} / \mathrm{CIM}<400 \mathrm{mg} * \mathrm{~h} / \mathrm{L}$. También fue posible relacionar valores de concentraciones plasmáticas pico de vancomicina en el rango de 28-35 $\mu \mathrm{g} /$ $\mathrm{mL}$ con $\mathrm{ABC} 24 \mathrm{~h} / \mathrm{CIM}>400 \mathrm{mg} * \mathrm{~h} / \mathrm{L}$. Finalmente, en el grupo de niños bajo 2 años de edad existió una relación lineal entre las concentraciones plasmáticas basales, las concentracionespico, el $\mathrm{Cl}$ de vancomicina y el $\mathrm{ABC}$ $24 \mathrm{~h} / \mathrm{CIM}$.

Los pacientes con patología crítica presentan una respuesta inflamatoria sistémica que altera los parámetros $\mathrm{PK} / \mathrm{PD}$, determinando un $\mathrm{Vd}$ real mayor al Vd teórico, influyendo en las concentraciones plasmáticas obtenidas de vancomicina ${ }^{19}$. A esto se suman situaciones clínicas que incluyen intervenciones terapéuticas y condiciones fisiológicas del paciente que aumentan el Vd (uso de fármacos vasoactivos, cargas de volumen con cristaloides o coloides e incremento del clearance de creatinina) ${ }^{20}$.

A pesar de obtener valores promedio de parámetros $\mathrm{PK} / \mathrm{PD}$ terapéuticos en la población critica pediátrica de nuestro estudio, se evidenciaron diferencias significativas cuando se determinó el número de pacientes que alcanzaron ABC 24 h/CIM > 400 mg*h/L. La mitad de 


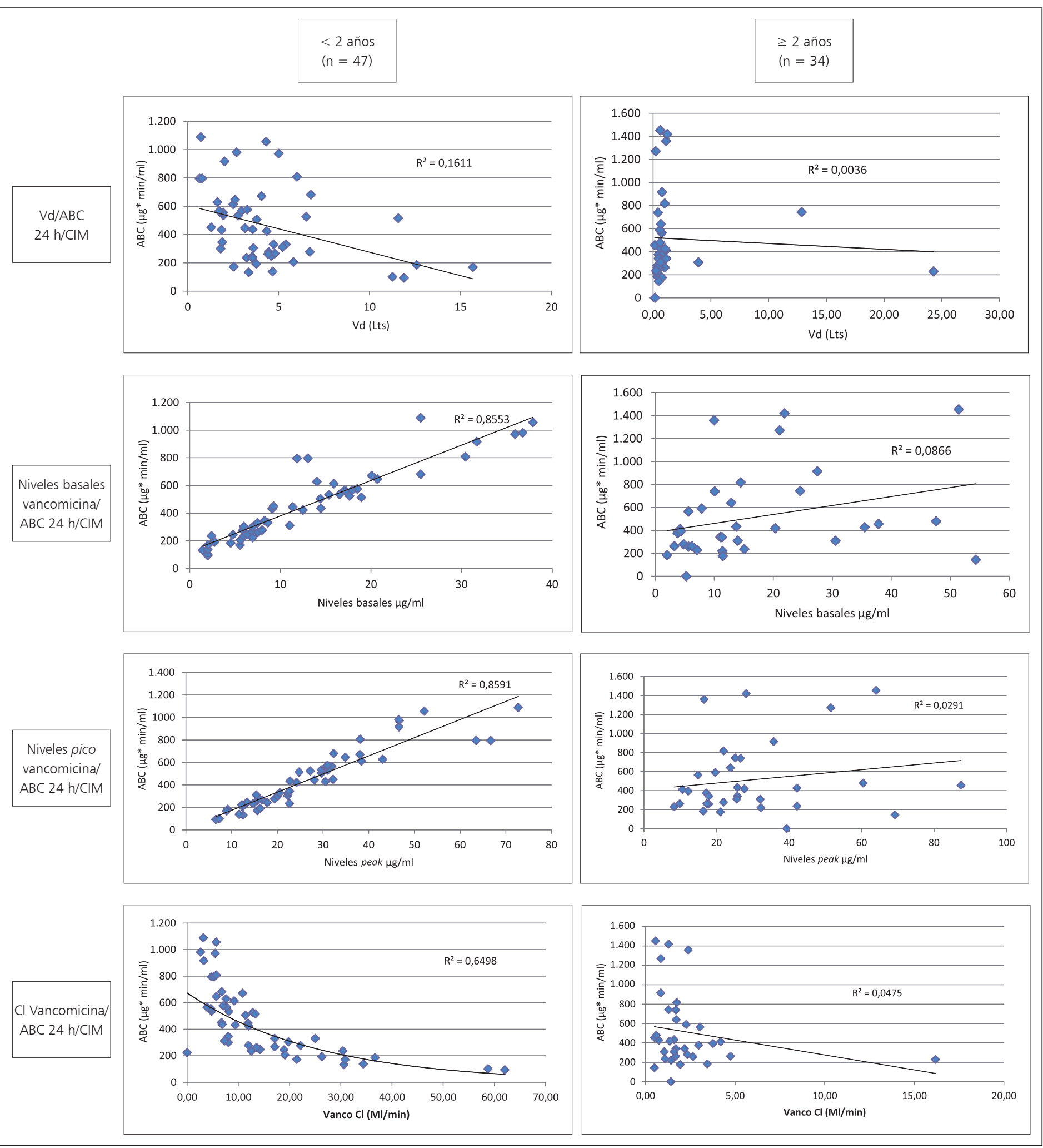

Figura 2. Relación de parámetros PK/PD: volumen de distribución, concentraciones plasmáticas basales y pico de vancomicina, clearance de vancomicina versus área de bajo la curva en niños bajo 2 años y sobre 2 años de edad hospitalizados en una Unidad de Paciente Crítico Pediátrico que recibieron tratamiento con vancomicina; vd: volumen de distribución; Cl: clearance; $\mathrm{ABC} 24$ h/CIM: área bajo la curva de exposición del fármaco en 24 h sobre la concentración inhibitoria mínima (CIM). 
nuestros pacientes no obtuvo un valor óptimo deseado, lo que podría relacionarse con una menor eficacia clínica del tratamiento con vancomicina ${ }^{21}$.

La importancia de la respuesta clínica en relación a parámetros óptimos de $\mathrm{PK} / \mathrm{PD}$ se ha demostrado en la literatura médica. Frymoyer y cols. ${ }^{22}$, estudió niños con infecciones por $S$. aureus que recibieron dosis de vancomicina de $40 \mathrm{mg} / \mathrm{kg} /$ día y $60 \mathrm{mg} / \mathrm{kg} /$ día. En este último grupo se obtuvo un mayor número de pacientes con $\mathrm{ABC}$ $24 \mathrm{~h} / \mathrm{CIM}>400 \mathrm{mg}{ }^{*} \mathrm{~h} / \mathrm{L}$. Eiland y cols. ${ }^{23}$, comunicaron que en pacientes que recibieron dosis convencionales de 40 a $60 \mathrm{mg} / \mathrm{kg} /$ día de vancomicina, 78\% alcanza concentraciones plasmáticas terapéuticas usando un rango de referencia de $5-15 \mu \mathrm{g} / \mathrm{mL}$ y sólo $49 \%$ si se considera un rango terapéutico de 10-20 $\mu \mathrm{g} / \mathrm{mL}$. De acuerdo a una ecuación predictora, estimaron que se requerirían dosis de vancomicina de $70 \mathrm{mg} / \mathrm{kg} /$ día para alcanzar concentraciones plasmáticas basales de al menos $10 \mu \mathrm{g} / \mathrm{mL}$.

Reconocer y comprender que los pacientes críticos pediátricos que padecen un shock séptico (oncológicos con neutropenia y fiebre, quemados, aquellos que han sido sometidos a trasplantes, pacientes con infecciones graves, en general) presentan alteraciones de los parámetros $\mathrm{PK} / \mathrm{PD}$, es fundamental, porque permite individualizar el tratamiento y optimizar el resultado clínico y microbiológico. El rango terapéutico de las concentraciones plasmáticas basales de vancomicina se ha definido entre 5 y $15 \mu \mathrm{g} / \mathrm{mL}^{18,21}$. En este estudio se encontraron diferencias significativas en ABC $24 \mathrm{~h} / \mathrm{CIM}$ entre pacientes con valores basales entre 5,0 y $9,9 \mu \mathrm{g} / \mathrm{mL}$ y aquellos con concentraciones plasmáticas $>10 \mu \mathrm{g} / \mathrm{mL}$, ya que en el rango terapéutico tradicional $(5-15 \mu \mathrm{g} / \mathrm{mL})$, las ABC $24 \mathrm{~h} / \mathrm{CIM}$ son significativamente $<400 \mathrm{mg} * \mathrm{~h} / \mathrm{L}$, aumentando el riesgo de falla terapéutica (84\% de los pacientes en esta serie). Estos resultados son comparables con los comunicados en el estudio de Piro y cols. ${ }^{24}$, quienes describieron la dosificación de vancomicina en pacientes pediátricos de unidades de oncología y trasplante de precursores hematopoyéticos con neumonía por $S$. aureus, demostrando que, con dosis de $40 \mathrm{mg} / \mathrm{kg}$ /día, las concentraciones plasmáticas basales de vancomicina entre 5-9 $\mu \mathrm{g} / \mathrm{mL}$ fueron predictoras de un menor éxito clínico y microbiológico. Nuestro estudio mostró que cuando los pacientes obtienen valores basales de vancomicina entre 10 y $15 \mu \mathrm{g} / \mathrm{mL}, 81 \%$ de ellos alcanza valores de ABC $24 \mathrm{~h} / \mathrm{CIM}>400 \mathrm{mg} * \mathrm{~h} / \mathrm{L}$.

Dado estos hallazgos, recomendamos al menos obtener concentraciones plasmáticas basales de vancomicina $>10$ $\mu \mathrm{g} / \mathrm{mL}$, lo cual se relacionaría con una mayor probabilidad de eficacia terapéutica ${ }^{21}$. En modelos como el analizado en nuestro estudio, la concentración plasmática pico de vancomicina fue importante en las determinaciones de ABC $24 \mathrm{~h} / \mathrm{CIM}>400 \mathrm{mg} * \mathrm{~h} / \mathrm{L}$, pudiendo demostrarse una correlación lineal en el grupo de pacientes bajo 2 años de edad. Cuando las concentraciones plasmáticas pico de vancomicina estuvieron en rangos de 28-35 $\mu \mathrm{g} / \mathrm{mL}$, se obtuvo un valor de ABC $24 \mathrm{~h} / \mathrm{CIM}>400 \mathrm{mg} * \mathrm{~h} / \mathrm{L}$, por lo que tomar ambas concentraciones plasmáticas (basal y pico) a las $48 \mathrm{~h}$ de iniciado el tratamiento con vancomicina nos entrega la ventaja de estimar ambos modelos PK/PD de análisis para las distintas especies gram positivas que pueden desencadenar infecciones graves.

Nuestro estudio tiene varias limitaciones. Los pacientes enrolados no tuvieron una categorización de gravedad (puntaje PRISM o PIM), pese a que todos ellos requirieron hospitalizarse en una UPCP. También es importante mencionar que para la medición de las concentraciones plasmática se utilizó la técnica de inmuno ensayo de polarización de fluorescencia (TDxFLx, Abbott Laboratories, IL, E.U.A.); hoy se tiende a la medición de niveles séricos por equipos de High Perfomance Liquid Cromathographie (HPLC) que son más precisos en sus resultados y ha provocado una nueva interpretación de los valores de corte de $\mathrm{ABC} 24 \mathrm{~h} / \mathrm{CIM}$ generando disminución del valor de $400 \mathrm{mg} \mathrm{x} \mathrm{h/L}$. Así mismo, no fue parte de este estudio determinar el éxito clínico y microbiológico asociado a los parámetros $\mathrm{PK} / \mathrm{PD}$ del paciente, lo que sin duda debe ser el paso siguiente a seguir para establecer la relevancia clínica de estas determinaciones. Para cumplir este objetivo, es indispensable realizar un estudio prospectivo, que incluya el registro de las variables clínicas y farmacológicas.

En conclusión, los niños que ingresan a la UPCP pueden estar expuestos a dosis sub-terapéuticas de vancomicina si no se individualiza el tratamiento según parámetros $\mathrm{PK} / \mathrm{PD}$. Es posible que niños con patología crítica debieran comenzar con dosis de vancomicina $>40$ $\mathrm{mg} / \mathrm{kg} /$ día y evaluar las concentraciones plasmáticas basales y pico a la hora 48 para obtener una terapia con mayor probabilidad de éxito terapéutico en cada paciente. Las dosificaciones estándares de vancomicina no entregan una seguridad respecto a la dosis clínicamente efectiva en el tratamiento, por lo que recomendamos realizar el monitoreo de concentraciones plasmáticas/séricas de vancomicina de manera sistemática en la UPCP para aumentar el éxito clínico y microbiológico. Serán necesarios estudios prospectivos de asignación aleatoria en población pediátrica para corroborar nuestros hallazgos.

Agradecimientos: Al equipo de enfermería de la UPCP del Hospital Luis Calvo Mackenna, al personal técnico del Laboratorio de Bioquímica por potenciar los estudios de farmacocinética y a los médicos de la UPCP.

\section{Resumen}

Introducción: El monitoreo farmacocinético-farmacodinámico $(\mathrm{PK} / \mathrm{PD})$ de vancomicina podría optimizar el manejo de niños que reciben vancomicina y se encuentran 
en shock séptico grave. Objetivo: Estudiar PK/PD de vancomicina en niños hospitalizados en una unidad de paciente crítico, relacionando parámetros farmacocinéticos (PFC) tradicionales con ABC $24 \mathrm{~h} / \mathrm{CIM}$. Material y Método: Estudio retrospectivo, descriptivo, en la Unidad de Paciente Crítico Pediátrico (UPCP) del Hospital Luis Calvo Mackenna en el período enero de 2008 - marzo de 2010. Se incluyeron niños $<18$ años tratados con vancomicina por sospecha/confirmación de infección estafilocóccica y recibieron dosis de $40 \mathrm{mg} / \mathrm{kg} /$ día. Se midieron concentraciones plasmáticas pico y basales. Los PFC calculados fueron realizados con software de farmacocinética TDMS ${ }^{\circledR}$. Resultados: Se enrolaron 84 niños. En relación del parámetro ABC 24h/CIM obtenido, $54 \%(45 / 84)$ de los niños alcanzaron niveles óptimos ( $>400 \mathrm{mg}^{*} \mathrm{~h} / \mathrm{L}$ ). Del análisis por PFC tradicionales, 49\% de los casos (41/84) presentó concentraciones basales de vancomicina en rango terapéutico $(5-15 \mu \mathrm{g} / \mathrm{mL})$ y de ellos, sólo 39\% (16/41) presentó un ABC 24 h/CIM > 400 $\mathrm{mg} * \mathrm{~h} / \mathrm{L}$. Todos los pacientes con concentraciones basales $\geq 15 \mu \mathrm{g} / \mathrm{mL}$ alcanzan un ABC $24 \mathrm{~h} / \mathrm{CIM}>400 \mathrm{mg} * \mathrm{~h} / \mathrm{L}$. Discusión: Los pacientes pediátricos que ingresan a la UPCP podrían estar expuestos a dosis sub-terapéuticas de vancomicina. Es recomendable individualizar el tratamiento de vancomicina utilizando monitoreo con parámetros $\mathrm{PK} / \mathrm{PD}$.

\section{Referencias bibliográficas}

1.- Brierley J, Carcillo J A, Choong K, Cornell T, Decaen A, Deymann A. Clinical practice parameters for hemodynamic support of pediatric and neonatal septic shock: 2007 update from the American College of Critical Care Medicine. Critical Care Medicine 2009; 37 (2): 666-88.

2.- Dellinger R, Carlet J, Masur H, Gerlach H, Calandra T, Cohen J, et al. Surviving sepsis campaign guidelines for management of severe sepsis and septic shock. Crit Care Med 2004; 32 (3): 858-73.

3.- Di Giantomasso D, Bellomo R, May C N. The haemodynamic and metabolic effects of epinephrine in experimental hyperdynamic septic shock. Intensive Care Med 2005; 31 (3): 454-6.

4.- Roberts J, Lipman J. Antibacterial dosing in intensive care. Clin Pharmacokinet 2006; 45 (8): 755-73.

5.- Di Giantomasso D, May C, Bellomo R. Norepinephrine and vital organ blood flow during experimental hyperdynamic sepsis. Intensive Care Med 2003; 29 (10): 1774-81.

6.- Power B M, Forbes A M, van Heerden P V, Ilett K F. Pharmacokinetics of drugs used in critically ill adults. Clin Pharmacokinet 1998; 34 (1): $25-56$

7.- Nicolau D. Optimizing outcomes with antimicrobial therapy through pharmacodynamic profiling. J Infect Chemother 2003; 9 (4): 292-6.

8.- Gunderson B W, Ross G H, Ibrahim K H,
Rotschafer J C. What do we really know about antibiotic pharmacodynamics. Pharmacotherapy 2001; 21 (11s): 302-18.

9.- Golper T A, Noonan H M, Elzinga L, Gilbert D, Brummett R, Anderson J L, et al. Vancomycin pharmacokinetics, renal handling, and nonrenal clearances in normal human subjects. Clin Pharmacol Ther 1988; 43: 565-70.

10.- Skhirtladze K, Hutschala D, Fleck T, Thalhammer F, Ehrlich M, Vukovich T, et al. Impaired target site penetration of vancomycin in diabetic patients following cardiac surgery. Antimicrob Agents Chemother 2006; 50: 1372-5.

11.- Rybak M. The pharmacokinetic and pharmacodynamic properties of vancomycin. Clin Infect Dis 2006; 42 (Suppl 1): S35-9.

12.- Moise-Broder P, Forrest A, Mary C. Pharmacodynamics of vancomycin and other antimicrobials in patients with Staphylococcus aureus lower respiratory tract infections. Clin Pharmacokinet 2004; 43 (13): 925-42.

13.- Moise-Broder P, Sakoulas G, Eliopoulos G M, Schentag J J, Forrest A, Moellering R C Jr. Accessory gene regulator group II polymorphism in methicillin resistant Staphylococcus aureus is predictive of failure of vancomycin therapy. Clin Infect Dis 2004; 38: 1700-5.

14.- Geraci J. Vancomycin. Mayo Clin Proc 1977; 52: 631-4.

15.- Sakoulas G, Gold H, Cohen R, Venkataraman L, Moellering R C, Eliopoulos G M. Effects of prolonged vancomycin administration on methicillin-resistant Staphylococcus aureus
(MRSA) in a patient with recurrent bacteraemia. J Antimicrob Chemother 2006; 57: 699-704.

16.- Mohr J, Murray B. Point: Vancomycin is not obsolete for the treatment of infection caused by methicillin-resistant Staphylococcus aureus. Clin Infect Dis 2007; 44: 1536-42.

17.- Giachetto G A, Telechea H M, Speranza N, Oyarzún M, Nanni L, Menchaca A. Vancomycin pharmacokinetic-pharmacodynamic parameters to optimize dosage administration in critically ill children. Pediatr Crit Care Med 2011; 12 (6): e250-4.

18.- Rybak M, Boike S. Monitoring vancomycin therapy. Drug Intell Clin Pharm 1986; 20 : 757-61.

19.- Roberts J. Pharmacokinetic issues for antibiotics in the critically ill patient. Crit Care Med 2009; 37: 840-51.

20.- Udy A. Augmented renal clearance implications for antibacterial dosing in the critically ill. Clin Pharmacokinet 2010; 49 (1): 1-16.

21.- Dehority W. Use of vancomycin in pediatrics, concise review of pediatrics infectious diseases. Pediatr Infect Dis J 2010; 29 (S5): 462-4.

22.- Frymoyer A. Current recommended dosing of vancomycin for children with invasive methicillin-resistant Staphylococcus aureus infections is inadequate. Pediatr Infect Dis J 2009; 28: 398-402.

23.- Eiland L. Assessment of vancomycin dosing and subsequent serum concentrations in pediatric patients, Ann Pharmacother 2011; 45: 582-9.

24.- Piro C. Initial vancomycin dosing in pediatric oncology and stem cell transplant patients. J Pediatr Hematol Oncol 2009: 31 (1): 3-7. 